\title{
BMJ Open Using patient-reported outcome measures (PROMs) to promote quality of care in the management of patients with established kidney disease requiring treatment with haemodialysis in the UK (PROM-HD): a qualitative study protocol
}

\author{
Nicola Elzabeth Anderson, ${ }^{1,2}$ Melanie Calvert, ${ }^{1}$ Paul Cockwell, ${ }^{1,2}$ Mary Dutton, ${ }^{1,2}$ \\ Olalekan Lee Aiyegbusi, ${ }^{1}$ Derek Kyte ${ }^{1}$
}

To cite: Anderson NE, Calvert M, Cockwell P, et al. Using patientreported outcome measures (PROMs) to promote quality of care in the management of patients with established kidney disease requiring treatment with haemodialysis in the UK (PROM-HD): a qualitative study protocol. BMJ Open 2018;8:e21532. doi:10.1136/ bmjopen-2018-021532

- Prepublication history for this paper is available online. To view these files please visit the journal online (http://dx.doi. org/10.1136/bmjopen-2018021532).

Received 8 January 2018 Revised 24 July 2018 Accepted 22 August 2018

\section{Check for updates}

(c) Author(s) (or their employer(s)) 2018. Re-use permitted under CC BY-NC. No commercial re-use. See rights and permissions. Published by BMJ.

${ }^{1}$ Centre for Patient Reported Outcomes Research, University of Birmingham, Birmingham, UK ${ }^{2}$ Department of Renal Medicine, University Hospitals Birmingham NHS Foundation Trust, Queen Elizabeth Hospital, Birmingham, UK

Correspondence to

Dr Derek Kyte;

d.g.kyte@bham.ac.uk

\section{ABSTRACT}

Introduction Patients undergoing haemodialysis (HD) for end-stage kidney disease often report a poor quality of life (QoL) and identify that improving QoL has a higher priority for them than improvements in long-term survival. Research suggests that regular collection and usage of patient-reported outcome measures (PROMs) in patients with chronic conditions may reduce hospitalisation, improve QoL and overall survival. In the UK, despite increased use within research settings, PROMs have not been introduced into the routine clinical care for patients undergoing $\mathrm{HD}$. We report the protocol for 'Using patient reported outcome measures (PROMs) to promote quality of care in the management of patients with established kidney disease requiring treatment with haemodialysis in the UK-PROM-HD'. The study aim is to investigate the methodological basis for the use of routine PROMs assessment, particularly using electronic formats (ePROMs) within clinical and research settings, to maximise the potential of PROM use in the management of the care of this patient group.

Methods and analysis The project will use qualitative methodology to explore, by thematic analysis, the views, perceptions and experiences of patients receiving HD and members of the HD multidisciplinary team regarding the collection and use of PROMs in routine clinical care, particularly ePROMs. This will involve interviews with up to 30 patients or until saturation is achieved and three focus group sessions with approximately 18 members of the clinical team delivering care to this patient group, which will be interpreted broadly to include both professional and non-professional staff.

\section{INTRODUCTION}

The number of patients with end-stage kidney diseases that are being treated with renal replacement therapy (RRT) by dialysis
Strengths and limitations of this study

- The use of semistructured interviews and focus groups allows a detailed and in-depth examination of the issues surrounding the use and implementation of renal patient-reported outcome measures to promote high-quality care in haemodialysis settings.

- The iterative approach allows the data collection tools to develop and expand as new information emerges, and as the data are based on human experience, it is powerful and possibly more compelling than quantitative data on this topic.

- The qualitative data arising from this study are unlikely to be generalisable, but will inform future planned research in this area.

The role of the researcher within the process can affect participant responses.

or transplantation continues to grow. In the UK, in December 2015, 61256 patients were receiving RRT, with haemodialysis (HD) being used as the treatment modality for $41 \%^{1}$ : of these 25114 patients, the large majority receive in-centre HD (17.8\% hospital, $21.2 \%$ satellite), $2 \%$ receive home HD. ${ }^{1}$

Patients receiving maintenance HD often experience a significant symptom burden associated with their renal failure, both physical and mental, ${ }^{2-5}$ and outcomes in terms of quality of life (QoL) and survival remain poor, even when compared with oncology patients. ${ }^{67}$ Traditionally reported outcomes such as mortality and progression of kidney disease to end-stage kidney disease are established indicators of health, ${ }^{8}$ but it is increasingly recognised that these 'hard' outcomes 


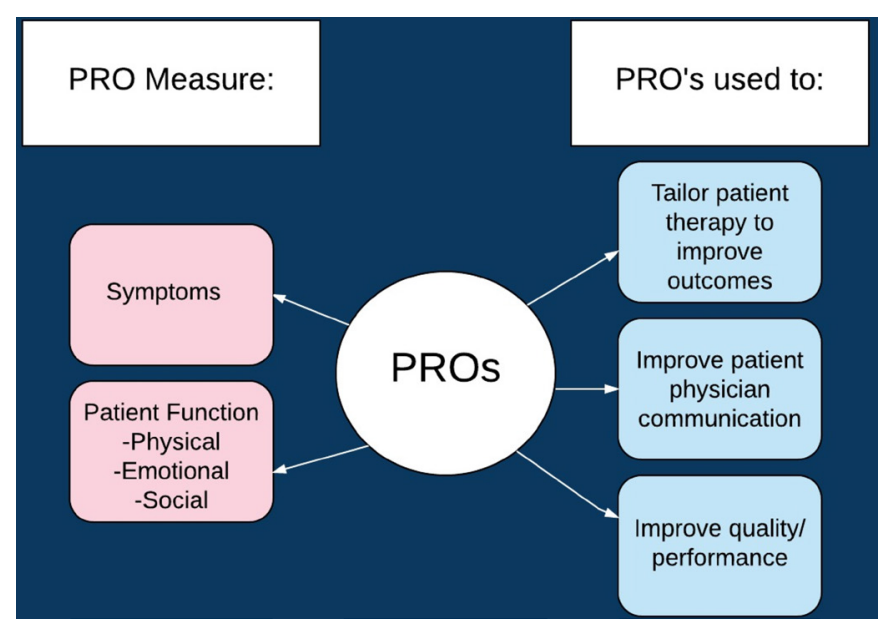

Figure 1 Conceptual framework for patient-reported outcome (PRO) integration in clinical care taken from Dobrozsi and Panepinto. ${ }^{21}$

do not adequately capture patients' overall QOL and may underestimate symptom burden. ${ }^{8}$ While biomedical outcomes, such as blood results, are often collected and reported during renal care and research, those outcomes which focus on well-being and QoL are often deemed more important by patients. ${ }^{10} 11$ Failure to address what matters to patients means symptoms and physical problems can go undetected and therefore untreated, ${ }^{2} 1213$ especially in-between clinic visits, ${ }^{9}$ and this leads to further suffering. Furthermore, patients approaching end-stage kidney disease may be willing to trade considerable life expectancy to reduce the burdens and restrictions associated with dialysis affecting QoL. ${ }^{14} 15$

UK health policy holds patient-centred care and shared decision making as a key tenet. ${ }^{16}$ Patient-centred care emphasises, and aligns treatment decisions with, patients' preferences, values and goals. ${ }^{17}$ This involves a change in the philosophy of care from a traditionally passive patient role to one where patients are actively involved in care planning and problem solving. ${ }^{18}$ With increased prominence being given to patient-centred care and shared decision making, the collection of QoL data and other patient-reported outcomes (PROs) are becoming progressively more important.

PROs are defined as 'any report of the status of a patient's health condition that comes directly from the patient, without interpretation of the patient's response by a clinician or anyone else'. ${ }^{19}$ Such PROs are most often collected using PRO measures (PROMs), self-completed questionnaires commonly collating data on general QoL and health-related QoL (HRQoL) as well as physical, emotional and social function, signs or symptoms, from the patients' own point of view. ${ }^{20}$ Evidence drawn from oncology studies suggests that PROMs can offer a standardised approach to measuring QoL and symptom burden, augmenting clinician understanding of the patient's health status and leading to overall improvements in patient care. ${ }^{21}$ The mode of administration of PROMs has traditionally been in paper format, but increasingly they are being completed electronically using various digital platforms (ePROMs).

Figure 1 outlines a conceptual framework for PRO integration in clinical care taken from Dobrozsi and Panepinto. $^{21}$

While the use of PROMs in routine nephrology practice is currently limited, their use in research as measures of clinical or treatment effectiveness is more widespread. ${ }^{8}$ However, there is no consensus on which PROMs to use or their implementation. As part of the international Standardised Outcomes in Nephrology InitiativeHaemodialysis (SONG-HD), major stakeholders have been consulted to help develop a core outcome set that reflects the priorities of patients, carers and clinicians for use in research trials involving HD. ${ }^{722}$ Patients and carers placed the highest value on outcomes which allowed them to maintain their daily well-being and lifestyle. These included the ability to travel, dialysis-free time and dialysis adequacy, which was conceptualised broadly as dialysis that is adequate for enabling patients to feel well, rather than attributable to a biochemical value. ${ }^{7}$

Initial feedback on the routine use of ePROMs received from the USA has focused on some of the perceived and experienced challenges in their use, such as increased time for completion and IT difficulties. However, with increased usage, evidence suggests PRO collection may not only enhance clinical care from the patients' perspective but may also improve physician satisfaction levels and prevent burnout. This is achieved by improvements in patient-clinician communication, which may increase workflow efficiency. ${ }^{23}$

While there is evidence to support the use of PROMs and particularly ePROMs in the management of patients diagnosed with other conditions, such as cancer, ${ }^{24-28}$ the evidence for the use and implementation of PROMs in the management of patients with end-stage kidney disease requiring treatment with HD is currently limited. However, although use of PROMs in nephrology research studies is increasingly common, standardisation in terms of how measures are chosen, implemented and reported is lacking. To help address these issues, this study aims to provide a rigorous exploration of the acceptability and operational feasibility of the collection of routine ePROM data with patients undergoing $\mathrm{HD}$, for potential use in clinical and research settings.

\section{Overall study aim}

To explore the feasibility and acceptability of using routine PROM assessment, in both clinical and research settings, in order to aid the management of patients with established renal failure undergoing HD.

\section{Key objectives and key associated questions}

1. To investigate the need for PROM use with patients and clinicians in HD settings.

- Do patients and clinicians feel PROM use would be beneficial? If so, how might their use alter practice and delivery of care? 
- Would answers to questions differ if PROMs are collected in a research setting, rather than for routine clinical practice?

2. To explore the practicalities associated with PROM implementation in HD settings.

- To assess the optimal timing of collection, that is, before or during dialysis sessions, from the patient and clinician perspective.

- To consider the optimal frequency of collection and to determine the average length of time required or that patients are prepared to spend on completion, from the patient and clinician perspective.

- To gauge the best setting for completion, that is, home or clinical area, from the patient and clinician perspective.

- To determine patients' ability to self-complete a questionnaire successfully and, if not, how much assistance they would require.

- To examine the feasibility of PROM collection in different patient groups (eg, older patients, patients of non-white ethnicity).

- To ascertain the most effective mode of administration for collection of PRO data, that is, in paper or electronic formats, such as mobile application or telephone recognition systems, in terms of usability and preferences for patients and clinicians.

- To explore the likely factors (barriers and facilitators) that may improve or discourage the completion of PROMs by patients and clinicians.

3. To explore the practicalities associated with PROM feedback in HD settings.

- To explore how patients would like to receive feedback from the clinical team regarding PRO data.

- To determine clinicians preferred method of displaying PRO data and the optimum way of providing patient feedback.

4. To explore the use of existing PROMs with patients in HD settings.

- To explore and determine whether existing validated PRO measurement tools for example, Kidney Disease Quality of Life-Short Form survey (KD-
QOL-SF), Kidney Disease Quality of Life-36-item survey (KDQOL-36), Integrated Palliative care Outcome Scale-Renal (IPOS-Renal) (see table 1) would be acceptable to patients undergoing HD and members of the renal multidisciplinary team (MDT) working within the HD setting for the routine monitoring of patient's health status.

- To evaluate the selected PRO questionnaires in terms of the relevance of their items and discuss any potentially missing items with patient and clinician participants.

\section{METHODS AND ANALYSIS \\ Project design}

This project will use qualitative methods, including semistructured interviews and focus groups, to explore the experiences and perceptions of patients undergoing HD and of clinicians within the MDT caring for these patients.

\section{Project setting}

University Hospitals Birmingham NHS Foundation Trust will be the host site for this project. Patients who are established on HD and are being treated within hospital and associated satellite dialysis units will be invited to participate in semistructured interviews to explore their views and perceptions on the routine use of PROMs. Members of the MDT caring for this patient group will be asked to participate in focus group sessions to explore their views on the use, administration and feedback of PROM data.

\section{Project participants}

Participants taking part in the patient interviews must be aged $\geq 18$ years old, be able to give valid informed consent and be conversant in everyday English. Only patients who have been established on HD $\geq 3$ months will be interviewed, allowing adjustment to specific issues associated with commencement of dialysis. Patients with an active intercurrent medical problem, which means they require enhanced routine clinical care, will be excluded from the study, such as patients who have experienced

Table 1 Description of questionnaires

\begin{tabular}{ll}
\hline Measure & Description \\
\hline $\begin{array}{l}\text { Kidney Disease Quality } \\
\text { of life-36 } \\
\text { (KDQOL-36) }\end{array}$ & $\begin{array}{l}\text { A 36-item health-related quality of life (HRQoL) measure designed for patients undergoing dialysis, } \\
\text { derived from the KDQOL-SF. } \\
\text { Three specific dimensions: } \\
\text { (1) signs and symptoms, (2) burden of kidney disease and (3) effects of kidney disease and a generic } \\
\text { core derived from the SF-12 (physical and mental scales). } \\
\text { Overall scores range from } 0 \text { to 100, with higher scores indicating better HRQoL }\end{array}$ \\
$\begin{array}{ll}\text { Kidney Disease Quality } \\
\text { of life-SF } \\
\text { (KDQOL-SF) }\end{array}$ & $\begin{array}{l}\text { An 80-item HRQoL measure designed for patients undergoing dialysis which includes the SF-36 } \\
\text { as a generic core (physical and mental scales) supplemented with eight kidney disease-targeted } \\
\text { dimensions and three additional QoL dimensions. } \\
\text { Scores range from } 0 \text { to } 100 \text { for each dimension with higher scores indicating better HRQoL }\end{array}$ \\
$\begin{array}{l}\text { Integrated Patient } 5657 \\
\text { Outcome Scale-Renal } \\
\text { (IPOS-Renal) }\end{array}$ & $\begin{array}{l}\text { IPOS-Renal is a short measure (11 questions), combining the most common symptoms renal } \\
\text { patients experience plus additional items from IPOS on concerns beyond symptoms, such as } \\
\text { information needs, practical issues, family anxiety }{ }^{33}\end{array}$ \\
\hline
\end{tabular}


a recent episode of acute kidney injury (within the last 3 months) or have a terminal illness that in the opinion of the consultant responsible for routine clinical care is likely to lead to the death of the patients within 6 months of study participation.

Purposive sampling will be undertaken. Participants will be selected for characteristics of relevance to the research questions. For example, sampling by ethnicity, age, gender and dialysis vintage will be conducted to elicit diverse experiences of the phenomenon of interest, PROM use in patients receiving HD (maximum variation sampling). Every effort will be made to recruit participants from minority ethnic groups to reflect the broad ethnic diversity within a large geographical dialysis catchment area that includes both rural and urban populations. Recruitment from more than one satellite HD unit will ensure that the study reflects the diversity which exists within the patient population. Data on participant characteristics will be collected as the study progresses in order to monitor sample diversity.

Participants invited to take part in MDT focus groups must be aged $\geq 18$ years old, be able to give consent and be a member of the MDT involved in the care of patients undergoing HD at the host site or an associated satellite HD unit. The MDT is viewed broadly; therefore, we will interview staff with and without membership of professional bodies and could include doctors, nurses, dietitians, physiotherapists, psychologists, healthcare assistants (HCAs) and unit housekeepers. While a highly technical clinical specialty, the role of renal HCAs continues to expand, and non- professional staff often foster unique and close relationships with patients. In other specialities, HCAs are being directly involved in the assessment of outcomes, ${ }^{29}$ and anecdotally, it is often stated that patients will share more, in terms of their experience of living with dialysis treatment, than with their nurses and doctors. Therefore, the views on the collection and implementation of renal PROMS by non-professional members of the MDT will be sought.

Based on experience from previous similar qualitative studies conducted by the research team, recruitment will continue until a target sample size of up to 30 patient participants is attained or until saturation is achieved. Saturation can be described as existing when no new codes, themes or categories are emerging from the data and the relationships between them can be explained. ${ }^{29}$

We aim to include up to 18 MDT participants in three focus groups (six participants in each group) and, if necessary, follow-up interviews will be conducted, depending on emerging data. The focus group sample size has also been derived from prior experience of such studies by the research team, and recruitment will continue until saturation is reached.

It is recognised that travel to and from dialysis units is a significant issue for patients receiving HD. If travel associated with routine $\mathrm{HD}$ appointments is disrupted or likely to be disrupted, reasonable travel expenses, that is, taxi fare to/from their home address, will be offered so that there is as little inconvenience as possible for the patient. Likewise, if focus groups are scheduled outside of staff members working hours, reasonable travel expenses will be made available.

\section{Recruitment methods}

A member of the renal research team will screen for potentially eligible patients using an in-house electronic patient records system at the host site, in collaboration with members of the dialysis care team. Eligibility will be confirmed by a clinician who is on the study delegation $\log$. Eligible patients will be sent a study-specific participant information sheet, which will be discussed further in clinic or prior to a dialysis session with a member of the research team, where willingness to participate can be ascertained. Ethical approval to post information to patients following screening but prior to consent has been obtained.

Members of the MDT will be approached about study participation, and if interested will be provided with a focus group information sheet. Those who would like to participate will be consented and invited to attend a focus group at a mutually convenient time.

Prior to interview or focus group attendance, participants will be sent copies of selected validated PROMs (KDQOL-36, KDQOL-SF and IPOS-Renal, see table 1) with instructions to read and review the contents so that they are familiar with the form and types of content PROMs can take. However, they will be advised that they do not need to complete these PROMs, and these are samples to assist discussion only.

Recent systematic reviews ${ }^{30}$ have assessed the quality of the measurement properties of existing PROMs and they recommend the KDQOL-36 for routine clinical practice and the slightly longer KDQOL-SF for clinical trials/ research purposes in dialysis patients. ${ }^{30}$

The UK Renal Registry, in response to the growing interest in PROMs by the renal community and as part of the 'Valuing Individuals' and 'Transforming Participation in Chronic Kidney Disease (TP-CKD)' programmes has begun piloting the collection of PROM data in the form of the generic EuroQol five dimension scale 5-level version (EQ-5D-5L) and the disease-specific Palliative care Outcome Scale - Symptoms - Renal (POS-S-Renal) questionnaires. ${ }^{32}$ The Palliative care Outcome Scale - Renal (IPOS-Renal) is a short measure that has been adapted from the POS-S-Renal and combines the most common symptoms experienced by renal patients with issues such as information needs, practical concerns, anxiety or low mood, family anxieties and overall feeling of being at peace. IPOS has been validated in a mixed population of those with cancer and non-cancer diagnosis, including renal patients, and shows good content and construct validity, reliability and responsiveness to change. ${ }^{33}$

The IPOS-Renal has been identified by the Renal Research Patient Advisory Group as brief and understandable and is currently undergoing content validation along with the KDQOL-36 in the local renal population 

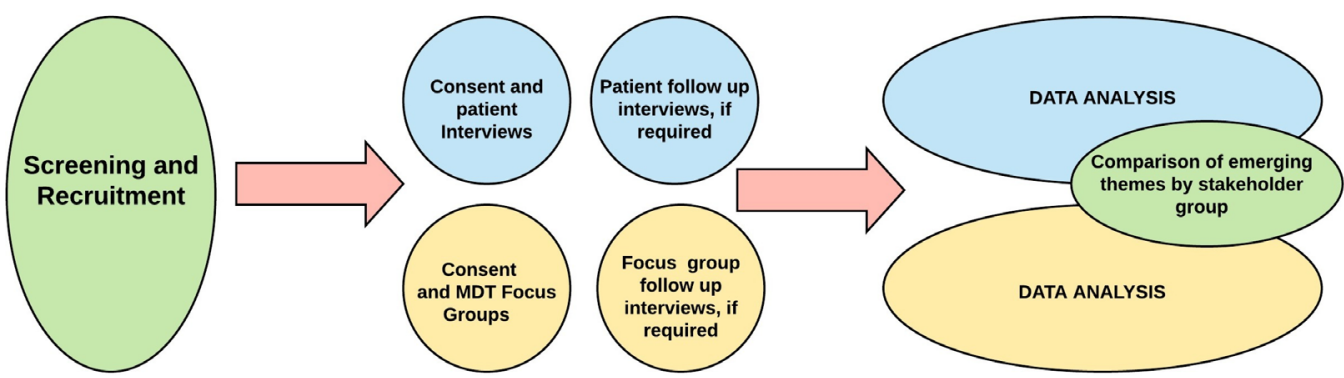

Figure 2 Study flow chart diagram. MDT, multidisciplinary team.

as part of the Using Patient-Reported Outcome Measures to promote quality of care and safety in the managment of patients with Advanced Chronic Kidney disease (PRO-TRACK) project. $^{30}$ Therefore, the KDQOL-36, KDQOL-SF and IPOS-Renal were selected as the PROMs that will be shown and discussed with patients and members of the MDT in this qualitative study. There is no charge for use from the developers for the KDQOL or IPOS-Renal. As the KDQOL-36 is a subset of the KDQOL-SF, patients will be asked to consider if the shorter PROM is adequate to assess the outcomes which matter most, compared with the broader, but longer and hence more time consuming to complete KDQOL-SF.

\section{Data collection}

The guiding qualitative research methodology chosen for this study is thematic analysis, as defined by Braun and Clarke. ${ }^{3455}$ Thematic analysis is a method for identifying, analysing and reporting patterns (themes) within data. It minimally organises and describes the data set in rich detail, also allowing interpretation of various aspects of the research topic. ${ }^{35}$ Patients will be interviewed faceto-face, or by telephone, to explore their experiences, perceptions and views of PROMs. The purpose of the focus groups will be to consider the content of selected questionnaires in current use with patients receiving HD and to discuss the various issues that relate to their use and administration. Face-to face or telephone interviews with selected staff may be conducted to explore their views further.

A topic guide will be used to structure the interviews so that all perceived relevant topics are discussed. However, there will be scope to digress from the guide if new and relevant topics are raised by participants. Analysis of transcripts will be conducted alongside data collection; this analysis may shape iterative adjustments to the topic guide to ensure all new and emerging topics are fully explored.

Figure 2 demonstrates the participant pathway through the study.

\section{Data analysis}

Analysis of the transcript data will be conducted using the Nvivo V.11 software package by QSR International.
Thematic analysis of the data will be conducted following the six-phase guide described by Braun and Clarke. ${ }^{34} 35$ The project team will randomly review a sample of coded transcripts for verification purposes. Data analysis will be carried out simultaneously with data collection and both will continue until no new themes emerge from the further analysis, that is, data saturation has been reached. ${ }^{36}$ Patient and MDT data will be initially analysed separately and then compared for recurrent or unique themes. Respondent validation also termed 'member checking' will be undertaken, whereby a summary of the main points arising from the interview will be sent to each participant for comments to explore the credibility of the results. ${ }^{37}$ In order to minimise the challenges associated with member checking, such as disagreement and distress, we will follow synthesised member-checking processes outlined by Birt et al. ${ }^{37}$

\section{Study withdrawal}

Study participants who are interviewed will be informed that they have the right to freely withdraw from the study, for any reason, at any time prior to their data being integrated into the data set (ie, within 5 working days following their interview). MDT study participants will be informed that they have the right to withdraw from the study, for any reason, before the focus group session. Participants will be reminded at the start of a session that, as the focus groups are audio recorded, it will not be possible to remove any of their subsequent data once the session has commenced due to the nature of evolving group discussion. It will be made clear to participants that they will not be required to supply a reason for their withdrawal and the decision will have no effect on either their care or terms of employment.

\section{Patient involvement}

The study protocol was developed in collaboration with members of the Renal Research Patients Advisory Group, Centre for Patient-Reported Outcomes Research, University of Birmingham and selected HD patients who gave comments and suggestions during the development of this project. 


\section{DISCUSSION}

The collection of symptom and QoL data through simple but validated questionnaires can potentially address the problems of underassessing symptom and QoL issues; recent studies suggested that ePROM collection may be sensitive enough to detect clinically relevant changes and could be used to provide 'early warning' prompts for intervention, ${ }^{38}$ enhancing and promoting patient engagement and activation in their treatment. ${ }^{3940}$ The use of ePROMs allows QoL and symptom data to be collected remotely and assessed and acted on in 'real time', ${ }^{30}$ with research demonstrating that patient reporting may detect potentially serious adverse event symptoms earlier than traditional clinician reporting. ${ }^{41}$

Furthermore, the use of PROMs has demonstrated substantial improvement in the recognition of problems which are traditionally underestimated by clinicians such as fatigue, pain, emotional and social health when compared with control groups. ${ }^{42}$ There is increasing evidence within oncology settings that the collection and feedback of PROMs can improve QoL, enrich the communication between clinician and patient and reduce hospitalisations and may improve overall survival. ${ }^{27} 2843$

The adequacy of a PROM depends on its characteristics and measurement properties; to be effective, a PROM must demonstrate reliability, construct and content validity and ability to detect change. ${ }^{30}$ However, to successfully implement the use of selected validated PROMs for routine monitoring of health status, it is important to consider a wide range of operational issues from both patient and clinician perspectives. These include: the optimal timing/frequency of PROM data collection, the feasibility of PROM collection in different patient groups (older patients, patients of non-white ethnicity) and the most effective mode of administration for collection of PROMs, that is, paper, electronic formats or telephone recognition systems.

It is important to consider appropriate recall period, and the need to be sure that the patient understands what is meant by the recall period. PRO instruments that cause patients to rely on their memory, that is, they need to recall over a long period of time or which compare their current state with an earlier period, or average their response over time, can possibly undermine content validity. Response is likely to be influenced by the patient's state at the time of recall, ${ }^{19}$ a factor which must be considered for patients who receive HD.

Results obtained using PROMs can vary according to the instructions given to patients or the training given to the interviewer or persons supervising PRO data collection during a clinical trial. ${ }^{19}$ There is every reason to assume this is true for PRO data collected outside trial settings, so it is important to consider how PROMs are presented and how clinicians are prepared in terms of delivery and feedback mechanisms.

What degree of respondent burden are HD patients prepared to tolerate? This will be affected by the length and regularity of proposed measures, ${ }^{44}$ formatting of the
PROM and questions that patients may feel unwilling or uncomfortable in answering; note there is always the proviso that patients may refuse to answer any questions, if they wish. Research suggests that patients with lower cognitive function are more likely to report higher response burden when completing PRO measures, ${ }^{45}$ and since we know that HD effects cognitive performance, ${ }^{46}$ it is important that burden is considered in this clinical setting.

Consideration needs to be given to the availability of privacy in the setting where the PROM is completed (eg, providing a private space for patients to complete or ask for assistance in completing questionnaires containing sensitive information about their sexual activity or substance abuse history, if required).

Most importantly, the timing of measurement needs to be considered, that is, 'when' in terms of a patient's dialysis regimen. Up to $70 \%$ of HD patients over 54 years of age may demonstrate cognitive impairment and it has been hypothesised that cognitive performance will be further impacted by the time point and place of testing. ${ }^{46}$ Such fluctuations may be relevant to PROM data collection. Data from a recent consensus meeting ${ }^{39}$ suggest PROs should preferably not be recorded during dialysis.

A shift from paper PRO collection to ePROMs is already evident in other settings. ${ }^{47}$ The use of ePROM capture and feedback needs to be investigated within the HD environment. Many HD satellite units are jointly run across the National Health Service and private sector, with established Electronic Patient Records (EPR) systems that could be used to incorporate PROs into their 'big data' collection. Small studies of home HD and Peritoneal Dialysis (PD) patients suggest that satisfaction with the use of tablet computers is high, even with those unused to this technology ${ }^{48}$ but the researchers identified challenges in the form of logistics of technology, security, institutional and financial support and electronic design. ${ }^{49}$ Such issues will be further explored. However, while qualitative methodology will allow exploration of preferences regarding mode of administration and issues around technical usability, it is noted that such methodology will not explore differences in scores or reliability by mode.

It is important that feedback mechanisms are also considered. Inadequate feedback means that outcomes reporting will not lead to improvements in patient care. By facilitating identification of HRQoL concerns and providing a structured way to discuss these, more clinical actions are then taken in response to these identified needs leading to more responsive and holistic care. ${ }^{50}$ There is limited evidence on the feedback of PROMs data to MDTs rather than individual doctors and nurses. Modern healthcare means it is unlikely that you will see the same clinician in a healthcare outpatient setting, although it is arguable that regular dialysis sessions do offer a degree of continuity compared with other specialities. However, it is suggested that PROMs can further facilitate MDT decision-making by focusing the team on aspects of care central to the patient. ${ }^{51}$ This aspect of 
PROM use will be explored further during the MDT focus group sessions.

It is important to consider how operational issues may differ in a research setting: would patients and clinicians be prepared to undertake a more burdensome regime of PRO data collection and how would feedback mechanisms and electronic symptom alerts to identify adverse events be managed? While the focus of this study is around views and perceptions on the acceptability and feasibility of routine collection of PROMs in a clinical setting, it is important that any nuances around collection in a research setting be gathered.

While the evidence for PRO data collection is becoming clearer, challenges remain, in the form of how the data are collected, interpreted and fed back to patients, both within and without research settings. The collection of PRO data may involve a shift in workflow, with QoL and symptom data potentially being reported in 'real-time' by patients into their electronic records. There needs to be effective mechanisms to respond to these data that are not excessively costly or time-consuming. ${ }^{43}$ The key to successful implementation is 'buy-in' from patients and clinicians and this requires collaboration with all stakeholders.

The results from this study will be used to inform further research on the implementation of PROMs in research and clinical settings, allowing extrapolation to other dialysis modalities. As a form of preliminary exploration, qualitative research is invaluable in shaping the development of an intervention by exploring its feasibility, acceptability and appropriateness. It is hoped this project will contribute to the development and implementation of PROMs that could enhance QoL assessment and hence improve overall QoL for individual patients requiring $\mathrm{HD}$ as well as improving service delivery and patient care.

\section{ETHICS AND DISSEMINATION}

This project was approved. It is planned that project findings will be presented at conferences and submitted for dissemination via peer-reviewed open access publications. Participants will be sent a summary of study findings, if they wish.

Acknowledgements The authors would like to thank all members of the Renal Research Patients Advisory Group (RRPAG), Centre for Patient-Reported Outcomes Research (CPROR), University of Birmingham and selected haemodialysis patients for comments and suggestions during the development of this project.

Contributors DK is the guarantor for this project. The project was conceived by DK, MC, PC, MD and NEA. NEA drafted the protocol manuscript. The manuscript was reviewed by DK, MC, PC, MD and OLA. The final draft was approved by all authors.

Funding This project is funded by the Queen Elizabeth Kidney Patients Association (QEKPA): an independent charity working to provide patients with information and support with renal matters.

Disclaimer The views expressed are the author's own and not an official position of the institution or funder. The researchers are independent from the funders.

Competing interests None declared.

Patient consent Not required.
Ethics approval The Gwasanaeth Moeseg Ymchwil (Wales 7) REC(17/WA/0281) following proportionate review and Health ResearchAuthority approval on 16 October 2017.

Provenance and peer review Not commissioned; externally peer reviewed.

Open access This is an open access article distributed in accordance with the Creative Commons Attribution Non Commercial (CC BY-NC 4.0) license, which permits others to distribute, remix, adapt, build upon this work non-commercially, and license their derivative works on different terms, provided the original work is properly cited, appropriate credit is given, any changes made indicated, and the use is non-commercial. See: http://creativecommons.org/licenses/by-nc/4.0/.

\section{REFERENCES}

1. MacNeill SJ, Ford D. UK renal registry 19th annual report: chapter 2 UK renal replacement therapy prevalence in 2015: national and centre-specific analyses. Nephron 2017;137(Suppl 1):45-72.

2. Scherer JS, Combs SA, Brennan F, et al. Sleep disorders, restless legs syndrome, and uremic pruritus: diagnosis and treatment of common symptoms in dialysis patients. Am J Kidney Dis 2017;69:117-28.

3. Lowney AC, Myles HT, Bristowe K, et al. Understanding what influences the health-related quality of life of hemodialysis patients: a collaborative study in England and Ireland. J Pain Symptom Manage 2015;50:778-85

4. Kraus MA, Fluck RJ, Weinhandl ED, et al. Intensive hemodialysis and health-related quality of life. Am J Kidney Dis 2016;68:S33-S42.

5. Theofilou P. Quality of life in patients undergoing hemodialysis or peritoneal dialysis treatment. J Clin Med Res 2011;3:132-8.

6. Wyld M, Morton RL, Hayen A, et al. A systematic review and metaanalysis of utility-based quality of life in chronic kidney disease treatments. PLoS Med 2012;9:e1001307.

7. Evangelidis N, Tong A, Manns B, et al. Developing a set of core outcomes for trials in hemodialysis: an international delphi survey. Am J Kidney Dis 2017;70:464-75.

8. Aiyegbusi OL, Kyte D, Cockwell P, et al. A patient-centred approach to measuring quality in kidney care: patient-reported outcome measures and patient-reported experience measures. Curr Opin Nephrol Hypertens 2017;26:442-9.

9. Pakhomov SV, Jacobsen SJ, Chute CG, et al. Agreement between patient-reported symptoms and their documentation in the medical record. Am J Manag Care 2008;14:530-9.

10. Nissenson AR. Improving outcomes for ESRD patients: shifting the quality paradigm. Clin J Am Soc Nephrol 2014;9:430-4.

11. Moss AH, Davison SN. How the ESRD quality incentive program could potentially improve quality of life for patients on dialysis. Clin $\mathrm{J}$ Am Soc Nephrol 2015;10:888-93.

12. Weisbord SD, Fried LF, Mor MK, et al. Renal provider recognition of symptoms in patients on maintenance hemodialysis. Clin J Am Soc Nephrol 2007;2:960-7.

13. Claxton RN, Blackhall L, Weisbord SD, et al. Undertreatment of symptoms in patients on maintenance hemodialysis. $J$ Pain Symptom Manage 2010;39:211-8.

14. Morton RL, Tong A, Howard K, et al. The views of patients and carers in treatment decision making for chronic kidney disease: systematic review and thematic synthesis of qualitative studies. BMJ 2010;340:c112.

15. Morton RL, Snelling $P$, Webster AC, et al. Factors influencing patient choice of dialysis versus conservative care to treat end-stage kidney disease. CMAJ 2012;184:E277-E283.

16. Richards T, Coulter A, Wicks P. Time to deliver patient centred care. BMJ 2015;350:h530.

17. Davison SN, Levin A, Moss AH, et al. Executive summary of the KDIGO Controversies Conference on Supportive Care in Chronic Kidney Disease: developing a roadmap to improving quality care. Kidney Int 2015;88:447-59.

18. Coates V. Role of nurses in supporting patients to self-manage chronic conditions. Nurs Stand 2017;31:42-6.

19. Food and Drug Administration, 2009. Guidance for industry. Patient reported outcomes measaures_Use in medical product development to support labelling claims. https://www.fda.gov/downloads/drugs/ guidances/ucm193282.pdf (accessed Dec 2017).

20. Hjollund NH, Larsen LP, Biering K, et al. Use of Patient-Reported Outcome (PRO) measures at group and patient levels: experiences from the generic integrated PRO System, WestChronic. Interact J Med Res 2014;3:e5.

21. Dobrozsi S, Panepinto J. Patient-reported outcomes in clinical practice. Hematology Am Soc Hematol Educ Program 2015;2015:501-6. 
22. Urquhart-Secord R, Craig JC, Hemmelgarn B, et al. Patient and caregiver priorities for outcomes in hemodialysis: an international nominal group technique study. Am J Kidney Dis 2016;68:444-54.

23. Rotenstein LS, Huckman RS, Wagle NW. Making patients and doctors happier - the potential of patient-reported outcomes. N Engl J Med 2017;377:1309-12.

24. Jensen RE, Snyder CF, Abernethy AP, et al. Review of electronic patient-reported outcomes systems used in cancer clinical care. $J$ Oncol Pract 2014;10:e215-e222.

25. Bennett AV, Jensen RE, Basch E. Electronic patient-reported outcome systems in oncology clinical practice. CA Cancer J Clin 2012;62:337-47.

26. Basch E, Snyder C. Overcoming barriers to integrating patientreported outcomes in clinical practice and electronic health records. Ann Oncol 2017;28:2332-3.

27. Basch E, Deal AM, Kris MG, et al. Symptom monitoring with patientreported outcomes during routine cancer treatment: a randomized controlled trial. J Clin Oncol 2016;34:557-65.

28. Basch E, Deal AM, Dueck AC, et al. Overall survival results of a trial assessing patient-reported outcomes for symptom monitoring during routine cancer treatment. JAMA 2017;318:197-8.

29. Seale C. The quality of qualitative research. London: Sage Publications, 2000

30. Aiyegbusi OL, Kyte D, Cockwell P, et al. Using Patient-Reported Outcome Measures (PROMs) to promote quality of care and safety in the management of patients with Advanced Chronic Kidney disease (PRO-trACK project): a mixed-methods project protocol. BMJ Open 2017;7:e016687.

31. Gibbons E, Fitzpatrick R. A structured review of patient reported outcome measures for adults with CKD. Oxford: University of Oxford, 2010.

32. (Undated) URRU. Overview of site and work Undated. https://www. england.nhs.uk/wp-content/uploads/2016/07/pa-uk-renal-registry. pdf

33. Palliative Care Outcome Scale, 2017. IPOS-Renal. https://pos-pal. org/maix/ipos-renal-in-english.php

34. Braun V, Clarke V. Succesful qualitative research. London: Sage Publications, 2013.

35. Braun V, Clarke V. Using thematic analysis in psychology. Qual Res Psychol 2006;3:77-101.

36. Fusch P, Ness L. Are we there yet? Data saturation in qualitative research. The Qualitative Report 2015;20:1408-16.

37. Birt L, Scott S, Cavers D, et al. Member Checking: A Tool to Enhance Trustworthiness or Merely a Nod to Validation? Qual Health Res 2016. doi: 10.1177/1049732316654870. [Epub ahead of print 22 Jun 2016].

38. Pittman ZC, John SG, Mclntyre CW. Collection of daily patient reported outcomes is feasible and demonstrates differential patient experience in chronic kidney disease. Hemodial Int 2017;21:265-73.

39. Breckenridge $\mathrm{K}$, Bekker HL, Gibbons $\mathrm{E}$, et al. How to routinely collect data on patient-reported outcome and experience measures in renal registries in Europe: an expert consensus meeting. Nephrol Dial Transplant 2015;30:1605-14.

40. Schougaard LM, Larsen LP, Jessen A, et al. AmbuFlex: tele-patientreported outcomes (telePRO) as the basis for follow-up in chronic and malignant diseases. Qual Life Res 2016;25:525-34.
41. Basch E, Bennett A, Pietanza MC. Use of patient-reported outcomes to improve the predictive accuracy of clinician-reported adverse events. J Natl Cancer Inst 2011;103:1808-10.

42. Detmar SB, Muller MJ, Schornagel JH, et al. Health-related qualityof-life assessments and patient-physician communication: a randomized controlled trial. JAMA 2002;288:3027-34.

43. Basch E. PRO's - Harnessing patient voices to improve clinical care. N Engl J Med 2017;376:105-8.

44. Senders A, Hanes D, Bourdette D, et al. Reducing survey burden: feasibility and validity of PROMIS measures in multiple sclerosis. Mult Scler 2014;20:1102-11.

45. Atkinson TM, Schwartz CE, Goldstein L, et al. How much is too much? predictors of patient response burden in the completion of patient-reported outcome assessments. Quality of Life Research 2017:26(Suppl 1):47-8.

46. Tholen S, Schmaderer C, Kusmenkov E, et al. Variability of cognitive performance during hemodialysis: standardization of cognitive assessment. Dement Geriatr Cogn Disord 2014;38:31-8.

47. eRAPID, 2017. eRAPID Towards safer delivery and monitoring of cancer treatments. Electronic patient self-Reporting of Adverseevents (AE): Patient Information and aDvice. http://pogweb.org/ index.php/erapid/

48. Schick-Makaroff $\mathrm{K}$, Molzahn A. Brief communication: patient satisfaction with the use of tablet computers: a pilot study in two outpatient home dialysis clinics. Can J Kidney Health Dis 2014;1:22.

49. Schick-Makaroff K, Molzahn A. Strategies to use tablet computers for collection of electronic patient-reported outcomes. Health Qual Life Outcomes 2015;13:2.

50. Etkind SN, Daveson BA, Kwok W, et al. Capture, transfer, and feedback of patient-centered outcomes data in palliative care populations: does it make a difference? A systematic review. J Pain Symptom Manage 2015;49:611-24.

51. Greenhalgh J. The applications of PROs in clinical practice: what are they, do they work, and why? Qual Life Res 2009;18:115-23.

52. Peipert JD, Hays RD. Methodological considerations in using patient reported measures in dialysis clinics. J Patient Rep Outcomes $2017 ; 1$.

53. Aiyegbusi OL, Kyte D, Cockwell P, et al. Measurement properties of patient-reported outcome measures (PROMs) used in adult patients with chronic kidney disease: a systematic review protocol. BMJ Open 2016:6:e012014

54. Peipert JD, Bentler PM, Klicko K, et al. Psychometric properties of the Kidney Disease Quality of Life 36-Item Short-Form Survey (KDQOL-36) in the United States. Am J Kidney Dis 2018;71.

55. Peipert JD, Bentler P, Klicko K, et al. Negligible impact of differential item functioning between Black and White dialysis patients on the Kidney Disease Quality of Life 36-item short form survey (KDQOL-36). Qual Life Res 2018:2699-707.

56. Aiyegbusi OL, Kyte D, Cockwell P, et al. Measurement properties of patient-reported outcome measures (PROMs) used in adult patients with chronic kidney disease: A systematic review. PLoS One 2017; 12:e0179733.

57. Hays RD, Kallich JD, Mapes DL, et al. Kidney Disease Quality of Life Short Form (KDQOL-SFTM) Version 1.3: A manual for use and scoring. Santa Monica, CA: Rand, 1995. Contract No.: P-7994. 